\section{Valor da equação Cockcroft-Gault na triagem de função renal reduzida em pacientes com hipertensão arterial sistêmica}

The value of Cockcroft-Gault equation for the screening of decreased renal function in patients with systemic arterial hypertension

\section{Autores}

Elisângela Milhomem dos Santos ${ }^{1}$

Ana Karina Teixeira da Cunha França ${ }^{2}$

João Victor Leal Salgado 3

Dyego José de Araújo

Brito $^{3}$

Isabela Leal Calado

Alcione Miranda dos

Santos $^{4}$

Natalino Salgado Filho ${ }^{5}$

${ }^{1}$ Programa de Pós-Graduação em Ciências da Saúde e Centro de Ciências, Saúde e Tecnologia da Universidade Federal do Maranhão -

UFMA.

${ }^{2}$ Departamento de Ciências

Fisiológicas da UFMA.

${ }^{3}$ Hospital Universitário da

UFMA

${ }^{4}$ Departamento de Saúde

Pública e Programa de

Pós-Graduação em Saúde

Coletiva da UFMA.

${ }^{5}$ Departamento de Medicina I

da UFMA.

Data de submissão: 14/02/2011 Data de aprovação: 18/07/2011

Correspondência para: Alcione Miranda dos Santos Rua Barão de Itapary, 155,

Centro

São Luís (MA) - Brasil

CEP: 65020-070

E-mail: amirandas@terra. com.br

Suporte financeiro: Fundação de Amparo à Pesquisa e do Desenvolvimento Científico e Tecnológico do Maranhão FAPEMA.

O referido estudo foi realizado na UFMA.

Os autores declaram a inexistência de conflitos de interesse.

\section{Resumo}

Introdução: A hipertensão arterial é um problema de saúde pública mundial e um dos principais fatores de risco para o desenvolvimento da doença renal crônica. Métodos: Com a finalidade de comparar a equação Cockcroft-Gault com a creatinina sérica e o clearance de creatinina $(\mathrm{ClCr})$ na triagem de função renal reduzida, realizouse um estudo transversal com 198 hipertensos de uma unidade básica de saúde. Foram analisados dados demográficos, nutricionais e clínico-laboratoriais. A função renal foi analisada pela creatinina sérica e pelo $\mathrm{ClCr}$ em urina de 24 horas. A taxa de filtração glomerular foi também estimada segundo a equação Cockcroft-Gault. Resultados: Os pacientes apresentaram idade média de 60,6 \pm 11,6 anos, e 73,7\% eram do sexo feminino. A prevalência de creatinina sérica $>1,2 \mathrm{mg} / \mathrm{dL}$ foi de 7,6\% e da taxa de filtração glomerular $<60 \mathrm{~mL} /$ minutos foi de $24,2 \%$, quando avaliadas pelo $\mathrm{ClCr}$ e pela equação Cockcroft-Gault. A filtração glomerular reduzida foi observada em homens mais velhos, com menor índice de massa corporal, valores normais de glicemia de jejum e maiores níveis de ácido úrico e pressão arterial sistólica. Discussão: A prevalência de função renal reduzida entre hipertensos varia consideravelmente dependendo da abordagem laboratorial utilizada. O clearance de creatinina, principalmente quando estimado pela equação de Cockcroft-Gault, mostrou ser um marcador mais acurado que a creatinina sérica na avaliação da taxa de filtração glomerular. Conclusões: A equação Cockcroft-Gault apresentou maior concordância com o clearance de creatinina, provando ser um confiável teste de triagem

\section{Abstract}

Introduction: Arterial hypertension is a worldwide public health problem and one of the major risk factors for chronic kidney disease development. Methods: In order to compare the CockcroftGault (CG) equation with serum creatinine and 24-hour creatinine clearance $(\mathrm{CrCl})$ for the screening of reduced renal function, a cross-sectional study of 198 hypertensive patients was undertaken at a basic health unit. The demographic, nutritional, and clinical laboratory data were analyzed. Renal function was assessed by serum creatinine and 24hour $\mathrm{CrCl}$. Glomerular filtration rate (GFR) was also estimated according to Cockcroft-Gault equation. Results: The patients had a mean age of $60.6 \pm 11.6$ years-old, and $73.7 \%$ were female. The prevalence of serum creatinine $>1.2$ $\mathrm{mg} / \mathrm{dL}$ was $7.6 \%$ and the prevalence of GFR $<60 \mathrm{~mL} / \mathrm{minute}$ was $24.2 \%$, when evaluated by the $\mathrm{CrCl}$ and $\mathrm{CG}$ equation. Reduced GFR was observed in older male patients, with lower body mass index, normal values of fasting blood glucose, and higher levels of serum uric acid and of systolic blood pressure. Discussion: The prevalence of decreased renal function among hypertensive patients varies considerably, depending on the laboratory investigation used. CG-estimated $\mathrm{CrCl}$ has shown to be more accurate than serum creatinine for assessing GFR. Conclusions: CG-estimated $\mathrm{CrCl}$ was highly similar to 24-hour $\mathrm{CrCl}$, proving to be a reliable primary care screening test for the early diagnosis of renal impairment in hypertensives. 
para o diagnóstico precoce e manejo de hipertensos com função renal reduzida na atenção básica.

Palavras-chave: Hipertensão. Falência renal crônica.

Taxa de filtração glomerular.
Keywords: Hypertension. Kidney failure, chronic. Glomerular filtration rate.

\section{INTRODUÇ̃̃O}

A hipertensão arterial (HA) configura-se como um dos maiores problemas de saúde pública no mundo. ${ }^{1}$ No Brasil, estima-se que mais de 30 milhões de indivíduos apresentam hipertensão ${ }^{1}$ e essa entidade representa um dos principais fatores de risco para doença renal crônica (DRC), sendo responsável por 35,8\% dos pacientes em terapia renal substitutiva (TRS). ${ }^{2,3}$

Estudos desenvolvidos com pacientes hipertensos revelaram prevalência de $22,3 \%$ de déficit de função renal, considerando a filtração glomerular (FG) $<60 \mathrm{~mL} /$ $\min . / 1,73 \mathrm{~m}^{2}$. Esses valores são bem superiores aos observados na população geral., ${ }^{4,5}$ Recentemente, a DRC adquiriu importância global em virtude do exponencial aumento dos casos registrados nas últimas décadas. O National Kidney Foundation ${ }^{6}$ aponta a necessidade urgente de políticas públicas voltadas para prevenção, diagnóstico precoce e intervenção de tal doença.

A identificação precoce e o manejo adequado da DRC têm sido reconhecidos como importantes medidas para retardar sua progressão. Na prática clínica, a avaliação inicial da função renal costuma ser realizada por meio da dosagem da creatinina plasmática. Essa conduta se deve à simplicidade do método; entretanto, já foi demonstrado que sua normalidade não é sinônimo da ausência de comprometimento da função renal e se mostra um parâmetro relativamente tardio para detecção da lesão. ${ }^{7}$

Outro parâmetro para avaliação da função renal é a determinação da FG por meio do clearance de creatinina em urina de 24 horas, que é a prova laboratorial mais utilizada, pois precede o aparecimento dos sintomas da doença. ${ }^{8} \mathrm{Na}$ impossibilidade do uso desse parâmetro e para superar a limitação da creatinina sérica, algumas fórmulas foram desenvolvidas para estimar a FG. As equações mais utilizadas são: equação de Cockroft-Gault ${ }^{9}$ e a derivada do estudo Modification of Diet in Renal Disease (MDRD). ${ }^{10} \mathrm{~A}$ equação de Cockcroft-Gault (CG) é uma das fórmulas preditivas mais empregadas para seguimento ambulatorial. ${ }^{11}$ Entretanto, essas equações ainda necessitam ser validadas em outras populações. O presente estudo comparou a equação CG com a creatinina sérica e o clearance de creatinina $(\mathrm{ClCr})$ como método na triagem de função renal reduzida em pacientes com HA sistêmica na atenção básica.

\section{Métodos}

Realizou-se um estudo transversal e analítico de janeiro a junho de 2008 com pacientes portadores de hipertensão primária, cadastrados no Programa HiperDia do Ministério da Saúde e em tratamento na Unidade Básica de Saúde da Vila Embratel, na cidade de São Luís do Maranhão. O estudo foi aprovado pelo Comitê de Ética do Hospital Universitário da Universidade Federal do Maranhão (HU-UFMA).

O tamanho da amostra foi calculado considerando uma população de 559 pacientes cadastrados no programa como hipertensos, sem diabetes associada, e a prevalência utilizada para alteração da função renal pela FG foi estimada em $22,3 \%$. $^{3}$ A margem de erro empregada foi de $4,0 \%$ e o nível de confiança de $95 \%$. Assim, a amostra resultou em 198 pacientes. Entretanto, para corrigir eventuais perdas durante o processo da coleta de dados, corrigiu-se a amostra em $10 \%$, totalizando 207 pacientes.

Neste estudo, foram incluídos pacientes hipertensos de ambos os sexos, com idade $\geq 20$ anos e que concordaram em participar. Como critérios de nãoinclusão, foram adotados: pacientes gestantes, portadores de diabetes, doença crônica consumptiva (câncer e AIDS), infecções no trato urinário ou estar em TRS.

A seleção dos pacientes foi realizada por sorteio aleatório, com reposição a partir de uma listagem dos hipertensos cadastrados no programa, que foi obtida na referida unidade de saúde. ${ }^{12}$ Os pacientes foram esclarecidos sobre o estudo e os que concordaram em participar receberam pessoalmente esclarecimento sobre o procedimento da coleta da urina de 24 horas, além das instruções por escrito e os recipientes para a coleta. Também foram orientados a comparecerem à Unidade de Saúde no dia da consulta de rotina, em jejum de 12 horas e com a urina de 24 horas coletada.

No dia da consulta, os pacientes responderam a um formulário estruturado contendo dados socioeconômicos, demográficos e a história clínica. A classificação econômica foi obtida de acordo com o 
Critério de Classificação Econômica Brasil (CCEB) e apresentada em classes. ${ }^{13}$ A escolaridade foi medida em anos de frequência escolar e categorizada em $\leq$ oito e $\geq$ nove anos. Considerou-se tabagista todo paciente que declarou ser fumante em algum momento da vida, independente da quantidade de cigarros, e etilista aquele que referiu consumo de bebida alcoólica no período da entrevista em qualquer quantidade e frequência.

No mesmo momento, aferiu-se a pressão arterial utilizando esfigmomanômetro digital $\left(\mathrm{Omron}^{\circledR}\right.$, modelo HEM 742) pelo método indireto, com o paciente sentado. Foram realizadas duas medições: a primeira no momento anterior ao preenchimento do formulário e a segunda, ao término. Foi considerada a média aritmética das duas medidas da pressão arterial. ${ }^{1}$

Em seguida, realizou-se a avaliação antropométrica. Foram aferidos peso (em quilogramas) em balança portátil digital $\left(\right.$ Plena $\left.^{\circledR}\right)$ e altura (em metros) em estadiômetro (Alturexata ${ }^{\circledR}$ ), seguindo técnicas preconizadas. ${ }^{14} \mathrm{O}$ índice de massa corporal (IMC) foi calculado por meio da razão do peso corporal e o quadrado da altura. A circunferência da cintura (CC) foi mensurada no ponto médio entre a última costela e a crista ilíaca, no momento da expiração, utilizando fita métrica não-extensível.

As amostras de sangue e urina (tipo I e de 24 horas) foram encaminhadas para posterior análise no Laboratório de Análises Clínicas do HU-UFMA. Para avaliação metabólica foram realizadas dosagens séricas de ácido úrico, colesterol total, triglicerídeos e glicemia em jejum pelo método enzimático; LDL colesterol (LDL-col) por meio da fórmula de Frieldwald; HDL colesterol (HDL-col) por meio de Roschlan et al. ${ }^{15}$ e a creatinina sérica pelo método cinético optimizado ultravioleta A proteinúria foi dosada em urina de 24 horas pelo método Bradford Modificado.

A função renal foi avaliada por meio da creatinina sérica, do clearance de creatinina dosado em urina de 24 horas e estimado pela equação de CG. ${ }^{9}$ Foi considerada alteração da função renal valores $>1,2 \mathrm{mg} / \mathrm{dL}$ para a creatinina sérica, ${ }^{16}<60 \mathrm{~mL} / \mathrm{min} . / 1,73 \mathrm{~m}^{2}$ para o clearance de creatinina e equação CG. ${ }^{6} \mathrm{O}$ clearance de creatinina dosado em urina de 24 horas foi analisado pelo método da imunoturbimetria. A estimativa do clearance de creatinina foi realizada pela equação de CG e corrigida para o sexo feminino. ${ }^{9}$ Quando identificada alteração da função renal, os pacientes foram encaminhados ao Ambulatório de Nefrologia do HU-UFMA para avaliação e tratamento com nefrologista.

As variáveis qualitativas são apresentadas por meio de frequências e porcentagens e as quantitativas por média e desvio padrão (média $\pm \mathrm{DP})$. Para avaliação da normalidade das variáveis quantitativas em estudo, realizou-se o teste Shapiro-Wilk. O teste $t$ de Student, quando aplicável, ou o teste de MannWhitney foram utilizados para comparar as variáveis quantitativas, segundo os critérios adotados para avaliar a função renal.

Para avaliar os fatores associados à função renal reduzida, utilizou-se o modelo de regressão logística uni e multivariado, tendo como variáveis independentes ácido úrico, pressão arterial sistólica (PAS) e idade. Foram considerados elevados níveis de PAS a partir de $140 \mathrm{mmHg}$ e de ácido úrico maiores do que $6 \mathrm{mg} / \mathrm{dL}$, para mulheres, e $7 \mathrm{mg} / \mathrm{dL}$, para homens. A variável idade foi categorizada como inferior a 60 anos e igual ou maior que 60 anos. Também foi calculada a razão de prevalência (RP) e seus respectivos intervalos de confiança de $95 \%$ (IC 95\%).

A concordância entre o clearance de creatinina dosado e estimado pela equação de CG foi avaliada por meio do modelo de regressão de Deming. A regressão de Deming é frequentemente aplicada na estimativa da reta entre um novo método e um de referência, considerando a existência de variância no novo método e no de referência. O referido método testa duas hipóteses: a de intercepto igual a 0 e a de inclinação da reta igual a 1 . Se as duas hipóteses são aceitas, isso significa que os dois métodos não diferem entre si. ${ }^{17} \mathrm{O}$ nível de significância adotado foi de $5 \%$. Os dados foram analisados no programa estatístico STATA 9.0.

\section{Resultados}

Dos 207 pacientes da amostra calculada, houve perda de nove indivíduos $(4,4 \%)$, sendo cinco por insuficiência de dados para avaliar a função renal e quatro por recusa em participar do estudo, totalizando uma amostra de198 hipertensos.

Os pacientes apresentaram idade média de 60,6 $\pm 11,6$ anos, com maior frequência do sexo feminino $(73,7 \%)$, possuíam menos que nove anos de frequência escolar $(83,8 \%)$ e estavam inseridos nas classes D e E (79,3\%), segundo o Critério de Classificação Econômica Brasil (CCEB). A prevalência de etilismo e tabagismo foi de 20,5 e $42,9 \%$, respectivamente (Tabela 1).

A prevalência de creatinina sérica $>1,2 \mathrm{mg} / \mathrm{dL}$ foi de $7,6 \%$. Prevalências iguais da TFG $<60 \mathrm{~mL} / \mathrm{min} . / 1,73 \mathrm{~m}^{2}$ $(24,2 \%)$ foram observadas quando avaliadas por meio do clearance de creatinina dosado e estimado pela equação CG (Tabela 2). 
Tabela 1 CARACTERÍSTICAS SOCIODEMOGRÁFICAS DE HIPERTENSOS EM TRATAMENTO

\begin{tabular}{|c|c|c|c|}
\hline Variáveis & $\mathrm{n}$ & $\%$ & Média \pm DP \\
\hline \multicolumn{4}{|l|}{ Sexo } \\
\hline Feminino & 146 & 73,7 & \\
\hline Masculino & 52 & 26,3 & \\
\hline Idade (anos) & 198 & & $60,6 \pm 11,6$ \\
\hline \multicolumn{4}{|l|}{ Classificação socioeconômica } \\
\hline Classes B2 e C & 41 & 20,7 & \\
\hline Classes D e E & 157 & 79,3 & \\
\hline \multicolumn{4}{|l|}{ Escolaridade (anos) } \\
\hline$\leq 8$ & 166 & 83,8 & \\
\hline$\geq 9$ & 32 & 16,2 & \\
\hline \multicolumn{4}{|l|}{ Tabagismo } \\
\hline Não & 113 & 57,1 & \\
\hline Sim & 85 & 42,9 & \\
\hline \multicolumn{4}{|l|}{ Etilismo } \\
\hline Não & 155 & 79,5 & \\
\hline Sim & 40 & 20,5 & \\
\hline Pressão arterial sistólica (mmHg) & 198 & & $150,0 \pm 23,7$ \\
\hline Pressão arterial diastólica (mmHg) & 198 & & $89,2 \pm 12,6$ \\
\hline Creatinina sérica (mg/dL) & 198 & & $0,9 \pm 0,3$ \\
\hline Cockcroft-Gault & 198 & & $80,3 \pm 30,6$ \\
\hline Clearance de creatinina & 198 & & $81,5 \pm 30,6$ \\
\hline
\end{tabular}

DP: desvio padrão.

Tabela 2 AVALIAÇÃo dA FUnÇÃo RENAL SEGUNDO CREATININA SÉRICA E CLEARANCE DE CREATININA DOSADO E ESTIMADO PELA EQUAÇÃO DE COCKCROFT-GAULT DE HIPERTENSOS EM TRATAMENTO

\begin{tabular}{lccc} 
Função renal & Creatinina sérica & Clearance creatinina & \multicolumn{2}{c}{ Cockcroft-Gault } \\
$\mathrm{n}(\%)$ & $\mathrm{n}(\%)$ & $150(75,8)$ \\
\hline Normal & $183(92,4)$ & $150(75,8)$ & $48(24,2)$
\end{tabular}

*Função renal reduzida: Creatinina sérica > 1,2 mg/dL e filtração glomerular $<60 \mathrm{~mL} / \mathrm{min} / 1,73 \mathrm{~m}^{2}$

A Tabela 3 apresenta as características demográficas, antropométricas e clínico-laboratoriais, considerando os critérios adotados para avaliação da função renal. De acordo com os valores de creatinina sérica, os hipertensos com função renal alterada se apresentaram mais idosos $(71,5 \pm 9,3$ versus $59,7 \pm 11,3$ anos; $\mathrm{p}=0,0002)$, do sexo masculino (12 versus $3 ; \mathrm{p}<0,0001)$, com menor IMC $(24,9$ $\pm 3,9$ versus $\left.27,6 \pm 4,9 \mathrm{~kg} / \mathrm{m}^{2} ; \mathrm{p}=0,0324\right)$ e valores menores de glicemia em jejum $(72,7 \pm 12,1$ versus $79,2 \pm 13,5 \mathrm{mg} / \mathrm{dL} ; \mathrm{p}=0,0294)$. Entretanto, possuíam maiores valores de PAS $(163,3 \pm 23,5$ versus $148,9 \pm 23,5 \mathrm{mmHg} ; \mathrm{p}=0,0218)$ e ácido úrico $(6,5 \pm 1,6$ versus $4,5 \pm 1,2 \mathrm{mg} / \mathrm{dL} ; \mathrm{p}<0,0001)$.

Considerando o clearance de creatinina dosado em urina de 24 horas, os pacientes que apresentaram função renal reduzida se revelaram mais velhos $(65,6 \pm 12,3$ versus $59,0 \pm 11,0$ anos; $\mathrm{p}=0,0007)$, com ácido úrico mais elevado $(5,0 \pm$ 1,6 versus $4,4 \pm 1,3 \mathrm{mg} / \mathrm{dL} ; \mathrm{p}=0,0305$ ) e com menores valores de IMC $(26,4 \pm 5,2$ versus $27,7 \pm 4,7$ $\left.\mathrm{kg} / \mathrm{m}^{2}\right)$ e proteinúria $(135,8 \pm 96,6$ versus $164,7 \pm$ 
Tabela 3 AVALIAÇÃO DAS CARACTERÍSTICAS DEMOGRÁFICAS, ANTROPOMÉTRICAS E CLÍNICO-LABORATORIAIS, SEGUNDO OS CRITÉRIOS ADOTADOS PARA AVALIAÇÃO DA FUNÇÃO RENAL DE HIPERTENSOS TRATADOS

\begin{tabular}{|c|c|c|c|c|c|c|}
\hline \multirow[b]{2}{*}{ Variáveis } & \multicolumn{2}{|c|}{ Creatinina } & \multicolumn{2}{|c|}{ Clearance de creatinina } & \multicolumn{2}{|c|}{ Cockcroft-Gault } \\
\hline & Normal & Alterada & Normal & Alterada & Normal & Alterada \\
\hline \multicolumn{7}{|l|}{ Sexo } \\
\hline $\mathrm{F}: M$ & $143: 40$ & $3: 12 * *$ & $114: 36$ & $32: 36$ & $114: 36$ & $32: 36$ \\
\hline Idade (anos) & $59,7 \pm 11,3$ & $71,5 \pm 9,3^{* *}$ & $59,0 \pm 11,0$ & $65,6 \pm 12,3^{* *}$ & $57,1 \pm 10,0$ & $71,5 \pm 9,3^{* *}$ \\
\hline Tempo HA (anos) & $7,8 \pm 7,6$ & $9,1 \pm 10,4$ & $8,2 \pm 8,1$ & $6,7 \pm 6,9$ & $7,5 \pm 6,3$ & $8,9 \pm 11,2$ \\
\hline IMC $\left(\mathrm{kg} / \mathrm{m}^{2}\right)$ & $27,6 \pm 4,9$ & $24,9 \pm 3,9^{*}$ & $27,7 \pm 4,7$ & $26,4 \pm 5,2^{*}$ & $28,5 \pm 4,7$ & $23,9 \pm 3,5$ \\
\hline $\mathrm{CC}(\mathrm{cm})$ & $94,6 \pm 11,9$ & $91,4 \pm 10,3$ & $94,8 \pm 11,1$ & $93,2 \pm 14,0$ & $96,7 \pm 11,2$ & $87,1 \pm 10,7^{* *}$ \\
\hline PAS (mmHg) & $148,9 \pm 23,5$ & $163,3 \pm 23,5^{*}$ & $149,1 \pm 24,0$ & $152,6 \pm 23,0$ & $147,8 \pm 23,1$ & $156,7 \pm 24,8^{*}$ \\
\hline PAD $(\mathrm{mmHg})$ & $89,2 \pm 12,8$ & $89,3 \pm 11,0$ & $89,8 \pm 12,8$ & $87,5 \pm 12,3$ & $90,3 \pm 12,5$ & $85,8 \pm 12,7^{*}$ \\
\hline Colesterol total (mg/dL) & $207,3 \pm 41,1$ & $203,9 \pm 26,0$ & $207,9 \pm 42,8$ & $204,4 \pm 30,2$ & $206,9 \pm 42,1$ & $207,3 \pm 33,6$ \\
\hline LDL-col (mg/dL) & $133,3 \pm 34,6$ & $131,5 \pm 25,6$ & $133,9 \pm 36,0$ & $130,9 \pm 26,8$ & $133,1 \pm 35,4$ & $133,3 \pm 29,4$ \\
\hline $\mathrm{HDL}-\mathrm{col}(\mathrm{mg} / \mathrm{dL})$ & $46,7 \pm 11,8$ & $44,0 \pm 10,1$ & $46,0 \pm 11,7$ & $48,1 \pm 11,5$ & $45,8 \pm 11,7$ & $48,9 \pm 11,2^{*}$ \\
\hline Triglicerídeos (mg/dL) & $135,9 \pm 69,6$ & $141,5 \pm 61,0$ & $139,3 \pm 67,4$ & $127,1 \pm 73,3$ & $139,7 \pm 72,9$ & $125,9 \pm 54,0$ \\
\hline $\mathrm{GJ}(\mathrm{mg} / \mathrm{dL})$ & $79,2 \pm 13,5$ & $72,7 \pm 12,1^{*}$ & $78,9 \pm 13,1$ & $78,2 \pm 14,8$ & $79,1 \pm 13,6$ & $77,3 \pm 13,2$ \\
\hline Ácido úrico (mg/dL) & $4,5 \pm 1,2$ & $6,5 \pm 1,6^{* *}$ & $4,4 \pm 1,3$ & $5,0 \pm 1,6^{*}$ & $4,4 \pm 1,3$ & $4,9 \pm 1,7^{*}$ \\
\hline Proteinúria (mg/24h) & $154,6 \pm 138,4$ & $194,0 \pm 148,7$ & $164,7 \pm 149,4$ & $135,8 \pm 96,6 * *$ & $157,8 \pm 148,1$ & $157,1 \pm 107,6$ \\
\hline
\end{tabular}

${ }^{*} p<0,05$; ${ }^{*} p<0,01$; F: feminino; M: masculino; HA: hipertensão arterial; IMC: índice de massa corporal; CC: circunferência da cintura; PAS: pressão arterial sistólica; PAD: pressão arterial diastólica; GJ: glicemia de jejum.

$149,4 \mathrm{~g} / 24$ horas; $\mathrm{p}=0,0090)$, como pode ser visto na Tabela 3

De acordo com a equação de CG, os pacientes com função renal diminuída se apresentaram também mais idosos $(71,5 \pm 9,3$ versus $57,1 \pm 10,0$ anos; $\mathrm{p}<0,0001)$, com maiores valores de PAS $(156,7 \pm 24,8$ versus $147,8 \pm 23,1 \mathrm{mmHg} ; \mathrm{p}=0,0201)$, HDL-col $(48,9 \pm 11,2$ versus $45,8 \pm 11,7 \mathrm{mg} / \mathrm{dL}$; $\mathrm{p}=0,0519)$ e ácido úrico $(4,9 \pm 1,7$ versus $4,4 \pm 1,3$ $\mathrm{mg} / \mathrm{dL} ; \mathrm{p}=0,0431)$ e com menores valores de CC $(87,1 \pm 10,7$ versus $96,7 \pm 11,2 \mathrm{~cm} ; \mathrm{p}<0,0001)$ e $\operatorname{PAD}(85,8 \pm 12,7$ versus $90,3 \pm 12,5 \mathrm{mmHg}$; $\mathrm{p}=0,0362)$ (Tabela 3).

Com relação aos fatores associados à função renal reduzida, avaliada pelo clearance de creatinina dosado, apenas ácido úrico alterado mostrou associação estatisticamente significante $(\mathrm{RP}=4,1$; IC95\% = 1,5 - 11,3). Pressão arterial sistólica acima de $140 \mathrm{~mm} / \mathrm{Hg}$ e idade $\geq 60$ anos não apresentaram associação. Quando avaliada a função renal pela equação de CG, nenhum dos fatores descritos foram estatisticamente significantes.

OmodeloderegressãodeDeming mostrou umacorrelação estatisticamente significante $(\mathrm{r}=0,45, \mathrm{p}<0,0001)$ entre o clearance dosado e o estimado pela equação de CG. O coeficiente angular da reta de regressão
Figura 1. Reta de regressão estimada: $y=-0,37+0,99 x$.

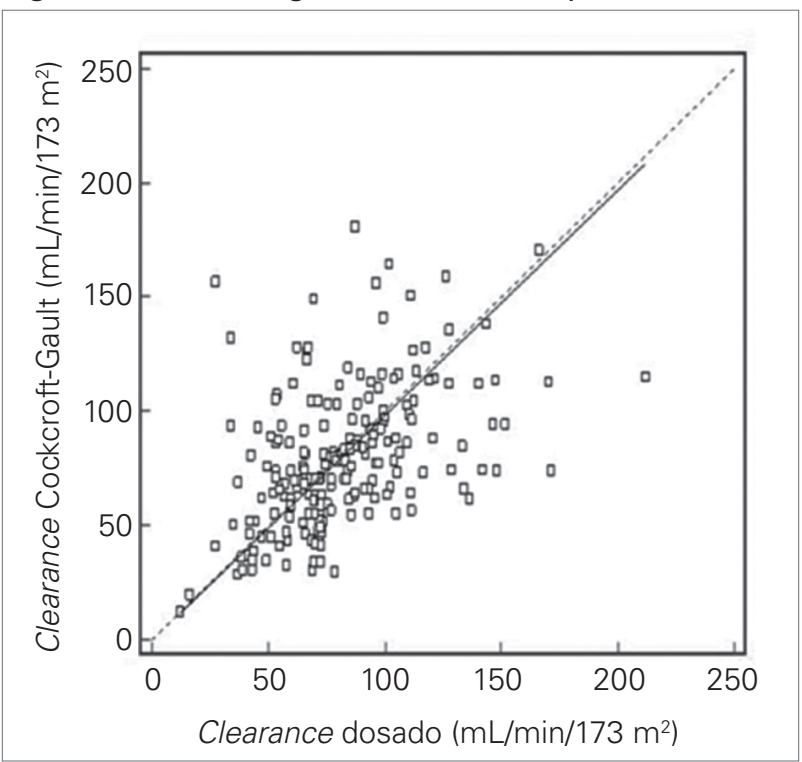

estimada foi igual a $0,99($ IC95\% $=0,64-1,33)$ e o intercepto $-0,37$ (IC95\% = -26,26 - 25,51). Na Figura 1 , observa-se que esta reta (a linha sólida no gráfico) não difere daquela com coeficiente angular igual a $1 \mathrm{e}$ intercepto zero (linha tracejada). Tal fato indica boa concordância entre o clearance dosado e o estimado pela equação de CG. 


\section{Dıscussão}

Neste estudo, encontrou-se prevalência de 7,6\% de creatinina sérica $>1,2 \mathrm{mg} / \mathrm{dL}$ e de $24,2 \%$ de FG reduzida $\left(<60 \mathrm{~mL} / \mathrm{min} . / 1,73 \mathrm{~m}^{2}\right)$, por meio da utilização dos marcadores do clearance de creatinina dosado e estimado pela equação CG. Esses achados corroboram com estudo realizado por Segura et al. ${ }^{3}$, os quais observaram igual prevalência de alteração da função renal pela creatinina sérica e 22,3 e $21,5 \%$ por meio do clearance de creatinina dosado e estimado pela equação de CG, respectivamente.

Entretanto, outros estudos de base populacional na Europ $\mathrm{a}^{18}$ e no Brasil, ${ }^{5}$ utilizando o clearance de creatinina, mostraram menor prevalência de taxa de FG reduzida em indivíduos da população geral $(6,9$ e $0,5 \%$, respectivamente). Esses achados ratificam que hipertensos apresentam maior comprometimento da função renal quando comparados com a população geral.

Outro aspecto relevante é a menor prevalência de alteração da função renal observada por meio da creatinina sérica. Isso pode ser atribuído ao fato de a creatinina sérica não se mostrar sensível para avaliação da função renal na DRC em pacientes assintomáticos, posto que só apresenta alteração da função renal após perda superior a $50 \%$ da FG. ${ }^{19}$ Deste modo, basear-se na creatinina sérica para avaliar a função renal pode protelar o diagnóstico, assim como a implementação de medidas que possam retardar a progressão da DRC. ${ }^{20}$

Neste estudo, a prevalência de alteração da função renal pelo clearance de creatinina dosado e estimado pela equação de $\mathrm{CG}^{9}$ foi semelhante e se revelou três vezes maior do que a alteração da função renal estimada pela creatinina sérica. $\mathrm{O}$ uso de fórmulas para determinar o clearance de creatinina tem sido amplamente utilizado e apresenta boa correlação com métodos radioisotópicos de determinação da FG, principalmente quando esta se apresenta diminuída $\left(<60 \mathrm{~mL} / \mathrm{min} . / 1,73 \mathrm{~m}^{2}\right) \cdot{ }^{10}$ Ademais, a utilização dessas equações para estimar a FG apresenta menor custo e maior praticidade na prática clínica, ${ }^{8-10}$ facilitando a triagem e o diagnóstico precoce da DRC em hipertensos na atenção básica, bem como o encaminhamento para o especialista.

Destaca-se que as fórmulas mais comumente utilizadas e conhecidas ${ }^{9} 10$ para estimativa do ritmo de FG foram validadas em estudos com populações-alvo. $\mathrm{O}$ clearance dosado em urina de 24 horas e o estimado pela equação de CG, realizados nesta pesquisa, obtiveram concordância estatisticamente significante.

A amostra em estudo se caracterizou pela predominância do sexo feminino (73,7\%), fato também observado em outros estudos com hipertensos. ${ }^{5,21,22}$ Freitas et al. ${ }^{23}$ sugerem que esse fato se deve à maior consciência das mulheres em relação a sua doença, buscando mais assistência à saúde. Ratificando esse achado, Boing e Boing $^{24}$ observaram em sua investigação maior número de mulheres inscritas no Programa HiperDia.

Considerando os métodos adotados para avaliação da função renal, os homens apresentaram maior frequência de creatinina sérica $>1,2 \mathrm{mg} / \mathrm{dL}$, sendo observada uma diferença significante quando comparados com as mulheres. Isso pode ser atribuído ao fato de a creatinina derivar do metabolismo da creatina e sua geração estar proporcional à massa muscular. ${ }^{10}$

A média de idade elevada $(60,6 \pm 11,6$ anos) observada neste estudo foi semelhante à encontrada por Didier e Guimarães. ${ }^{21}$ Este dado reflete a transição demográfica que a população brasileira vem vivenciando nas últimas décadas..$^{25}$ Ademais, evidências epidemiológicas demonstram que a prevalência de HA aumenta com a idade, estimando-se em $50 \%$ nas pessoas com mais de 60 anos. ${ }^{26}$ Nesta pesquisa, os pacientes que apresentaram função renal reduzida possuíam idade mais elevada do que aqueles com função renal normal. Outros estudos também observaram piora da função renal com a idade. ${ }^{3,5}$ Concordando com esse fato, Lindeman et al. ${ }^{27}$ chamam atenção para diminuição progressiva da FG na ordem de $1 \mathrm{~mL} / \mathrm{min}$./ano a partir dos 40 anos. Esse declínio pode em parte ser explicado pelo processo fisiológico normal do envelhecimento orgânico e celular e está associado com mudanças estruturais nos rins. Entretanto, os critérios para diagnóstico da DRC incluem um limiar absoluto não-ajustado para os efeitos da idade sobre os valores normais da FG estimada, o que tem gerado diagnósticos falso-positivos de DRC. ${ }^{28}$

A maioria dos hipertensos participantes do estudo possuía menos de nove anos de escolaridade $(83,8 \%)$ e pertencia às classes socioeconômicas mais baixas $(79,3 \%)$, retratando uma população por excelência pobre e com baixa instrução. Sturmer et al..$^{22}$ referem que pessoas que vivem em condições menos favorecidas apresentam menor probabilidade de receber manejo adequado para o tratamento da HA.

Em relação ao tabagismo, este estudo demonstrou a prevalência desse hábito em $42,9 \%$ dos pacientes avaliados. Estudos de Fuchs et al. ${ }^{29}$ e Sturmer et al..$^{22}$ revelaram prevalências similares, 51,8 e 47,4\%, respectivamente. Tal hábito de vida aumenta o risco para o declínio da função renal, ${ }^{30}$ recomendando-se, portanto, o seu combate.

Quanto ao consumo de bebida alcoólica, 20,5\% dos pacientes eram etilistas, considerando o consumo de qualquer quantidade e frequência de bebida alcoólica. Conceição et al..$^{31}$ observaram prevalência de 53,6\%, 
adotando-se o mesmo critério deste estudo. Fuchs et al..$^{29}$ encontraram $11,7 \%$ de etilistas, considerando-se o consumo diário de $30 \mathrm{mg}$ ou mais de etanol. Em função da diversidade do critério adotado para o etilismo em diversos estudos, esses apresentam discrepância nos achados. Alguns pesquisadores demonstraram que o consumo de bebida alcoólica está associado ao aumento dos níveis pressóricos ${ }^{32,33}$ e recomendam limitar ou abolir sua ingestão, com o intuito de auxiliar no controle da pressão arterial. ${ }^{1}$

Considerando os indicadores antropométricos, os pacientes com função renal diminuída apresentaram menor IMC quando comparados àqueles com função renal normal, sendo estatisticamente significante. $\mathrm{O}$ mesmo foi observado para a CC, porém a diferença significativa foi demonstrada apenas para o método CG. ${ }^{9}$ Esses achados podem ser justificados em consequência do avanço da DRC, no qual estudos demonstraram redução espontânea da ingestão de proteína com a diminuição da FG, aumentando assim o risco de desnutrição. ${ }^{34,35}$

Níveis elevados de HDL-col apresentaram significância estatística apenas no grupo de pacientes com declínio da função renal quando avaliados pela equação de CG. ${ }^{9}$ Tal achado difere do comumente encontrado na literatura. É importante ressaltar que este estudo não avaliou uso de medicação hipolipemiante, o que representa uma limitação.

A PAS, na análise univariada, apresentou-se mais elevada nos grupos com comprometimento da função renal avaliados pela creatinina sérica e equação de CG, ${ }^{9}$ quando comparada ao grupo de pacientes com função renal normal. No entanto, a PAD se apresentou menor nos pacientes com FG reduzida, quando avaliada pelo método CG. ${ }^{9}$

Os hipertensos com função renal comprometida apresentaram ácido úrico elevado, independente do método utilizado para avaliar a função renal. Outros estudos também encontraram níveis mais elevados de ácido úrico em pacientes com FG reduzida. ${ }^{36-38}$

Quando avaliou-se conjuntamente PAS, ácido úrico e idade, apenas o ácido úrico $(\mathrm{RP}=4,1$; IC95\% = 1,5 - 11,3) permaneceu associado com a FG reduzida. Shlipak et al. ${ }^{38}$ sugerem que a hiperuricemia identifica um estado de DRC préclínica que antecede ao desenvolvimento da doença e que está associado com desfechos adversos. Essa frequentemente se associa com a HA e é considerada um indicador sensível de lesão hipertensiva de órgãoalvo. ${ }^{39}$ Dessa forma, o ácido úrico é um marcador de doença renal, visto que o declínio da FG compromete, de maneira importante, sua excreção.
Os grupos apresentaram uma média elevada de proteinúria, sendo que o grupo com função renal reduzida, avaliada pelo clearance dosado, mostrou significância estatística quando comparada com os de função renal normal. A excreção de proteína pela urina é considerada preditor para desenvolvimento e agravamento da DRC, ${ }^{40}$ constituindo-se um elemento importante no diagnóstico e seguimento, pois quanto maior for a proteinúria, mais rápida é a perda da função renal. ${ }^{41}$

$\mathrm{O}$ presente estudo não utilizou um marcador padrão-ouro para avaliar precisamente a FG, devido ao alto custo, difícil acesso para pacientes em acompanhamento na atenção básica e elevado tempo requerido para o procedimento. Nesse contexto, o clearance de creatinina dosado é o método mais utilizado pelo seu baixo custo e por ser mais acessível aos usuários de serviço públicos. ${ }^{7}$ Outra limitação é a ausência de avaliação da terapia medicamentosa, que pode interferir na FG e no perfil lipídico. No entanto, diante da análise dos resultados, conclui-se que os pacientes hipertensos com função renal diminuída eram idosos, apresentaram menor IMC, maiores níveis de PAS, ácido úrico e proteinúria. Foi evidenciado que a creatinina sérica se mostrou um marcador tardio para avaliação da função renal, e o clearance de creatinina estimado pela equação CG apresentou forte correlação com o dosado. Esse achado demonstra a importância da equação CG na triagem de hipertensos com comprometimento da função renal, posto que revelou-se um marcador mais precoce do que a creatinina, com baixo custo e facilidade de utilização na atenção básica.

\section{Agradecimentos}

Os autores são gratos ao Conselho Nacional de Desenvolvimento Científico e Tecnológico $(\mathrm{CNPq})$ e à Fundação de Amparo à Pesquisa e ao Desenvolvimento Científico e Tecnológico do Maranhão (FAPEMA), pelo apoio financeiro ao projeto, ao Hospital Universitário Presidente Dutra e à Unidade de Saúde da Vila Embratel e aos pacientes que aceitaram participar do estudo.

\section{ReferÊnCIAS}

1. Sociedade Brasileira de Nefrologia. VI Diretrizes Brasileiras de Hipertensão Arterial. J Bras Nefrol 2010;32:1-64.

2. Sociedade Brasileira de Nefrologia. Censo geral 2008. [cited 2008 out 10]. Disponível em: http://www.sbn. org.br/ Censo/2008/censoSBN2008.pdf.

3. Segura J, Campo C, Ruilope LM, Rodicio JL. How prevalent and frequent is the presence of mild renal insufficiency in essential hypertension? J Ciln Hypertens 2002;4:332-6. 
4. USRDS. Annual Data Report. Atlas of End-Stage Renal Disease in the United States, Bethesda. USA: National Institute of Diabetes and Digestive and Kidney Diseases; 2001.

5. Passos VMA, Barreto SM, Costa-Lima MFF. Detection of renal dysfunction base on serum creatinine levels in a Brazilian community. The Bambuí Health and Ageing Study. Braz J Med Biol Res 2003;36:393-401.

6. National Kidney Foundation - NKF. K/DOQI clinical practice guidelines for chronic kidney disease: evaluation, classification and stratification. Am J Kidney Dis 2002;39:1-266.

7. Salgado JV, Neves FA, Bastos MG, França AK, Brito DJ, Santos EM, et al. Monitoring renal function: measured and estimated glomerular filtration rates - a review. Braz J Med Biol Res 2010;43:528-36.

8. Bostom AG, Kronenberg F, Ritz E. Predictive performance of renal function equations for patients with chronic kidney disease and normal serum creatinina levels. J Am Soc Nephrol 2002;13:2140-44.

9. Cockcroft DW, Gault MH. Prediction of creatinine clearance from serum creatinine. Nephron 1976;16:3141.

10. Levey AS, Bosch JP, Greene T, Regers N, Roth DA. A more accurate method to estimate glomerular filtration rate from serum creatinine: a new prediction equation. Modification of Diet in Renal Disease Study Group. Ann Intern Med 1999;130:461-70.

11. Rigalleau V, Lasseur C, Perlemoine C, Barthe N, Raffaitin C, Chauveau P, et al. Cockcroft-Gault formula is biased by body weight in diabetic patients with renal impairment. Metabolism 2005;55:108-12.

12. França AKTC, Santos AM, Calado IL, Santos EM, Cabral PC, Salgado JVL, et al. Filtração glomerular e fatores associados em hipertensos atendidos na atenção básica. Arq Bras Cardiol 2010;94:779-87.

13. ABEP - Associação Brasileira de Empresas de Pesquisa. Critério de Classificação Econômica Brasil. ABEP, 2003. [cited 2008 apr 20]. Disponível em http://www. abep.org/codigosguias/ABEP_CCEB.pdf.

14. Kamimura MA, Baxmann A, Sampaio LR, Cuppari L. Avaliação Nutricional. In: Cuppari L. Guia de Nutrição: Nutrição Clínica no Adulto. São Paulo: Manole; 2005. p. 71-108.

15. Roschlan P, Bernt GW. Enzimatische Best immung desgesamtcholesterius in serum. J Clin.Chem.Bio 1974;12:403-407.

16. Di Napoli Filho M, Burmeister JE, Miltersteiner DR, Campos BM, Costa MG. Estimativa da função renal pela fórmula de Cockcroft e Gault em pacientes com sobrepeso ou obesidade. J Bras Nefrol 2008;30:18591.

17. Linnet K. Performance of Deming regression analysis in case of missespecified error ratio in method comparison studies. Clin Chem 1998;44:1024-31.

18. New JP, Middleton RJ, Klebe B, Farmer CKT, Lusignant $\mathrm{S}$, Stevens PE, et al. Assessing the prevalence, monitoring and management of chronic kidney disease in patients with diabetes compared with those without diabetes in general practice. Diabetic Medicine 2007;24:364-69.

19. Shemesh O, Golbetz H, Kriss JP, Meyers BD. Limitations of creatinine as a filtration marker in glomerulopathic patients. Kidney Int 1985;28:830-8.
20. Pinto PS, Silva FJ, Munch ECSM, Chaoubah A, Bastos RV, Andrade LCF, et al. Inadequabilidade da Creatinina Sérica na Identificação Precoce da Disfunção Renal. J Bras Nefrol 2004;26:196-201.

21. Didier MT, Guimarães AC. Otimização de Recursos no Cuidado Primário da Hipertensão Arterial. Arq Bras Cardiol 2007;88:218-24.

22. Sturmer G, Dias-da-Costa JS, Olinto MTA, Menezes $\mathrm{AMB}$, Gigante DP, Macedo S. O manejo não medicamentoso da hipertensão arterial sistêmica no Sul do Brasil. Cad Saúde Pública 2006;22:1727-37.

23. Freitas JB, Tavares A, Kohlmann O, Zanella MT, Ribeiro AB. Estudo Transversal sobre o Controle da Pressão Arterial no Serviço de Nefrologia da Escola Paulista de Medicina - UNIFESP. Arq Bras Cardiol 2002;79:117-22.

24. Boing AC, Boing AF. Hipertensão Arterial Sistêmica: o que nos dizem os sistemas brasileiros de cadastramentos e informações em saúde. Rev Bras Hipertens 2007;14:84-8.

25. Instituto Brasileiro de Geografia e Estatística - IBGE. Síntese de Indicadores Sociais: Uma análise das condições de vida da população brasileira - 2008 . [cited 2008 aug 22]. Disponível em: http://www.ibge. gov.br/home/estatistica/população/condicaodevida/ indicadoresminimos/sinteseindicsociais2008/indic_ sociais2008.pdf.

26. Whelton PK, He J, Appel LJ, Jefrey CA, Havas S, Kotchen TA, et al. Primary prevention of hypertension: clinical and public health advisory from the National High Blood Pressure Education Program. JAMA 2002;288:1882-8.

27. Lindemann RD, Tobin J, Shock NW. Longitudinal studies on the rate of decline in renal function with age. J Am Geriatr Soc 1985;33:278-85.

28. Glassock RJ, Winearls C. Ageing and the glomerular filtration rate: Truths and consequences. Trans Am Clin Climatol Assoc 2009;120:419-28.

29. Fuchs FD, Gus M, Moreira LB, Moraes RS, Wiehe M, Pereira GM, et al. Anthropometric Indices and the Incidence of Hypertension: A Comparative Analysis. Obesity Research 2005;13:1515-17.

30. Bleyer A, Shemanski L, Burke G, Hansen KJ, Appel RG. Tobacco, hypertension, and vascular disease: Risk factors for renal function decline in an older population. Kidney Int 2000;57:2072-9.

31. Conceição TV, Gomes FA, Tauil PL, Rosa TT. Valores de pressão arterial e suas associações com fatores de risco cardiovasculares em servidores da Universidade de Brasília. Arq Bras Cardiol 2006;86:26-31.

32. Okubo Y, Suwano Y, Kobayzschi E, Nogawa K. Alcohol consumption and blood pressure change: 5 year follow-up study of the association in normotensive workers. J Hum Hypertens 2001;15:367-72.

33. Souza ARA, Costa A, Nakamura D, Mocheti LN, Stenavato Filho PR, Ovando LA. Um estudo sobre hipertensão arterial sistêmica na cidade de Campo Grande, MS. Arq Bras Cardiol 2007;88:441-6.

34. Ikitzler TA, Greene JH, Wingard RL, Parker RA, Hakim RM. Spontaneous dietary protein intake during progression of chronic renal failure. J Am Soc Nephrol 1995;6:1386-91. 
35. Chertow GM, Hsu C, Johansen K. The Enlarging Body of Evidence: Obesity and Chronic Kidney Disease. J Am Soc Nephrol 2006;17:1501-2.

36. Bastos RMR, Teixeira MTB, Chaoubah A, Bastos RV, Bastos MG. Hiperuricemia: Um marcador para doença renal crônica pré-clínica? J Bras Nefrol 2009;31:32-8.

37. Tomita M, Mizumo S, Yamanaka H, Hosoda Y, Sakuma K, Matuoka Y, et al. Does hyperuricemia affect mortality? A prospective cohort stydy of Japanese male workers. J Epidemiol 2000;10:403-9.

38. Shlipak MG, Katz R, Sarnak MJ, Fried LF, Newman $\mathrm{AB}$, Stehman-Breen $\mathrm{C}$, et al. Cystatin $\mathrm{C}$ and prognosis for cardiovascular and kidney outcomes in elderly persons without chronic kidney disease. Ann Intern Med 2006;145:237-46.

39. Johnson RJ, Feig DI, Kang DH, Herrera-Acosta J. Resurrectin of uric acid as a causal risk factor for essential hypertension. Hypertension 2005;45:18-20.

40. Jafar TH, Stark PC, Schmid CH, Landa M, Maschio G, de Jong PE, et al. Progression of chronic kidney disease: the role of blood pressure control, proteinúria, and angiotensin-converting enzyme inhibition - a patientlevel meta-analysis. Ann Intern Med 2003;139:244-52.

41. Alves MAR. Diagnóstico da doença renal crônica: avaliação de proteinúria e sedimento urinário. J Bras Nefrol 2004;26:6-8. 